\title{
Comparing the performance of various filters on stoned kidney images
}

\author{
Pronab Kumar Mondal ${ }^{1}$, Mst. Maklina Khatun ${ }^{2}$, Umme Hadia Akter ${ }^{3}$ \\ ${ }^{I}$ (Dept. of Computer Science and Engineering/ Jatiya Kabi Kazi Nazrul Islam University, Bangladessh) \\ ${ }_{2}^{2}$ (Dept. of Computer Science and Engineering/ Jatiya Kabi Kazi Nazrul Islam University, Bangladessh) \\ ${ }^{3}$ (Dept. of Computer Science and Engineering/ Jatiya Kabi Kazi Nazrul Islam University, Bangladessh)
}

\begin{abstract}
Noise removing is the most important task for biomedical image analysis. Noise is always presents in ultrasound digital images during image acquisition. Filtering is a tool for noise removing. In this paper we compare the performance of four filters-Median Filter, Averaging/Mean Filter, Gaussian Filter, Wiener filter. This paper presents which filter is best for removing noise like Gaussian, Salt \& Pepper, Poisson, and Speckle noise from stoned kidney image. Which filter is best, is measured by calculating the MSE and PSNR of filtered image.
\end{abstract}

Keywords: Median Filter, Averaging/Mean Filter, Gaussian Filter, Wiener filter, Gaussian noise, Speckle noise, Ultrasound Image.

\section{Introduction}

Digital images are prone to a variety of types of noise. Noise is the result of errors in the image acquisition process that result in pixel values that do not reflect the true intensities of the real scene. Image processing is widely used in all fields like astronomy, space exploration, medical imaging, scanning techniques and many others. The information carried by digital images gets corrupted by impulse noise during their acquisition or transmission [1].The use of ultrasound imaging in medical diagnosis is well established because of its noninvasive nature, low cost, capability of forming real time imaging and continuing improvement in image quality [3]. One of the most important tasks in image processing is to suppress the noise from images which have been corrupted by different reasons such as imperfection of imaging system, bad focusing, motion and etc. The noise removal techniques could assist to present the more precious characteristics of images which are not well understood [4]. It would be useful in different applications of fields such as astronomy, forensic science and particularly in medical field which need more reliable techniques to get the accurate outcome. Since selecting the de-noising algorithm depends on the application, therefore, the knowledge of noises in an image is essential to choose the suitable de-noising algorithm [5]. Several median based techniques are studied extensively due to ease of implementation and efficiency in terms of noise removal and edge preservation as compared to their linear counterparts which cause image blurring [2]. The important property of image denoising model is that it should completely remove noise with preserving edges. Basically the image quality is measured by the peak signal to noise ratio (PSNR) and mean square error (MSE) [2]. However, in this paper first image is taken and some noise is added to image to make it as noisy image and then noisy image is decomposed by filters. It becomes very important to de-noise the image before applying to different applications [6]. The principle approach of image de-noising is filtering. Filters used to remove noise are averaging/mean filters, median filters, wiener filter, Gaussian Filter and Adaptive Wiener filter.

The algorithm of the proposed work is given below:

i. Acquisition of Ultrasound stoned kidney image.

ii. Adding different type of noises to the input kidney image.

iii. Applying different type of filters to noised kidney image.

iv. Calculate MSE (Mean Squared Error) PSNR (peak signal-to-noise ratio) for each type of filtering image.

v. Decide which filter is gives the best performance for removing noise from stoned kidney

image according to lowest MSE and highest PSNR.

\section{Types Of Noise}

Noise in image, is any degradation in an image signal, caused by external disturbance while an image is being sent from one place to another place via satellite, wireless and network cable.

Here we are discussing about four types of noise

2.1 Gaussian Noise: Gaussian noise is a kind of noise which influences all the pixel values. The random noise value ateach pixel of noisy image is gained through the Gaussian probability density function. The density function of this noise is defined as the following [9]. 
$F(g)=\frac{1}{\sqrt{2 \pi \sigma^{2}}} e^{-\frac{(g-m)^{2}}{2 \sigma^{2}}}$

$\mathrm{W}$ here $\mathrm{g}$ is a gray level; $\mathrm{m}$ is the average of the function and $\sigma^{2}$ is the variance of the noise.

\subsection{Salt and pepper noise}

Salt \& Pepper noise contains black and white spots in an image. This noise is usually formed by the errors in data transmission and image sensor. It is figured out from different experimental researches that most of camera's images results in discrete pulses; salt and pepper noise and zero mean the Gaussian noise [10].

Probability Density Function of salt and pepper noise is:

$$
F(g)=\left\{\begin{array}{l}
\left\{\begin{array}{l}
P_{a} g=a \\
P_{b} g=b \\
0, \text { otherwise }
\end{array}\right.
\end{array}\right.
$$

\subsection{Poisson noise}

Poisson noise (Shot noise) is a kind of electronic noise which arise along the paucity of photons. In other words, it happens when the confined number of particles which carry energy is sufficiently narrow to ascend the detectable statistical f fluctuations in a measurement [11].

\subsection{Speckle noise:}

Speckle noise as a multiplicative noise is caused by coherent processing of backscattered signals from multiple distributed objects. It is nearly arisen in different imaging systems like laser, acoustics and SAR (Synthetic Aperture Radar) imagery. Speckle noise enriches the mean grey level of a local area [12,2]. This noise follows a gamma distribution as:

$$
F(g)=\frac{g^{\alpha-1}}{(\alpha-1) a^{\alpha}} e^{-\frac{g}{a}}
$$

Where $\mathrm{a}$ is variance and $\mathrm{g}$ is gray level.

\section{Filtering Techniques}

Lot of filters are exists for removing noise from digital image. In this paper, removing the noise from kidney stone image, we use four filters and examine which filter is best for removing noise.

\subsection{Median Filter}

Median filter is one of the most popular and efficient filters which is simple to implement. Although the basic drawback of Median Filtering is blurring the image in process, it could preserve the edges while suppressing the noise as well [12,13, and 14]. Specifically, this filter supplants a pixel by the median of all pixels in the sliding window [14].

$$
\begin{gathered}
\hat{f}(x, y)=\operatorname{median}\{g(s, t)\} \\
(x, t) \in S_{x y}
\end{gathered}
$$

Median filtering is very effective at removing various kinds of noise. But it is special for "Salt and pepper noise" [15].

\subsection{Averaging/ Mean Filter}

Average filter or mean filter is simple and easy to understand. It performs smoothing of images (i.e. reducing variation of intensity between one pixel and the next). Mean filters have the simpler structure relative to Median filters. It replaces the value of every pixel in an image with the mean ('average') value of its neighbors[17].The behavior of this filter in presence of signal dependent noises is well [16]. Mean filter is usually used to suppress the small details in an image and also bridge the small gaps exist in the lines or curves [17]. The mean filter is defined as the following. 


$$
\begin{aligned}
& g(i, j)=\frac{1}{M \times N} \sum f(m, n) \\
& m=1,2 \ldots M, \quad n=1,2 \ldots N .
\end{aligned}
$$

Where $S$ is the neighborhood defined by the filter mask of the point $f(i, j)$, centered at point $f(i, j)$.

\subsection{Gaussian Filter}

Gaussian filter is a particular filter known for blurring and suppressing the noise [18]. This filter is a 2D convolution operator with the weights selected pursuant to the shape of Gaussian function .The function is defined as the following [19].

$$
g(x, y)=\frac{1}{M} \sum f(x, y) \exp \left[-\left((x-i)^{2}+(y-j)^{2}\right) / 2 \sigma^{2}\right] \quad(i, j) \in S
$$

Where $\mathrm{S}$ is every pixel set in the neighborhood.

And,

$$
M=\sum \exp \left[-\left((x-i)^{2}+(y-j)^{2}\right) / 2 \sigma^{2}\right]
$$

The equation defines the set of pixels and corresponding weights of S.

\subsection{Wiener Filter}

Wiener filter is a linear filter. It provides linear estimation of a desired signal sequence from another related sequence [8]. Wiener filter provide solution for stationary signals in finding signal estimation problems. It provides successful results in removing noise from images. Wiener filter is based on statistical approach. The Wiener filter purpose is to reduce the amount of noise present in a signal by comparison with an estimation of the desired noiseless signal. It is based on a statistical approach [20].

Wiener filters play a central role in a wide range of applications such as linear prediction, echo cancellation, signal restoration, channel equalization and system identification [21]. The function can be written by,

$$
f(u, v)=\left[\frac{H(u, v) *}{H(u, v)^{2}+\left[\frac{s n(u, v)}{s f(u, v)}\right.}\right] G(u, v)
$$

where $\mathrm{H}(\mathrm{u}, \mathrm{v})$ is the degradation function \& $\mathrm{H}(\mathrm{u}, \mathrm{v}) *$ is its conjugate complex and $\mathrm{G}(\mathrm{u}, \mathrm{v})$ is the degraded image. Functions $\operatorname{Sf}(\mathrm{u}, \mathrm{v})$ and $\operatorname{Sn}(\mathrm{u}, \mathrm{v})$ are power spectra of the original image and the noise [22].

\section{Performance Parameters}

The performance parameters are most important criteria to justify the simulation results of different filters used in stoned kidney image. Besides, the quality of the Images will also be measured by the traditional distortion measurements such as Mean Square Error (MSE) and Root Mean Square Error (RMSE), Peak Signal to Noise Ratio (PSNR) between the original images and the output images. The quality of de-noised image is measured by following parameters:

\subsection{Mean Square Error (MSE):}

The MSE is the cumulative square error between the De-noised image and the original image defined by:

$$
M S E=\frac{1}{M+N} \sum_{i=1}^{M} \sum_{j=1}^{N}[x(i, j)-y(i, j)]^{2}
$$

Where, $x(i, j)$ is original image and $y(i, j)$ is de-noised image. MSE should be as low as possible.

\subsection{Peak Signal to Noise Ratio (PSNR):}

PSNR is the ratio between maximum possible power of a pixel value and the power of distorting noise which affects the quality of the original image [12]. It is defined by: 


$$
P S N R=10 \log _{10}\left(R^{2} / M S E\right)
$$

Where $\mathrm{R}$ is maximum value of pixel present in an image and MSE is the mean square error between original and de-noised image with $\mathrm{M} * \mathrm{~N}$ size.

\section{Results and Analysis}

For our research work, we first converted acquired kidney stone image to gray scale kidney stone image, then adding different types of noises to input image then applying different types of filter to noisy image then we calculate MSE and PSNR for deciding which filter is best for removing noise from kidney stone image.

The following figures represent the sample of kidney stoned images after simulating the Gaussian, Salt \& Pepper, Speckle and Poisson noise, and de-noising the results using Median filter, Mean /average filter, Gaussian Filter and Wiener filter. The Simulation is run by MATLAB R2015a.

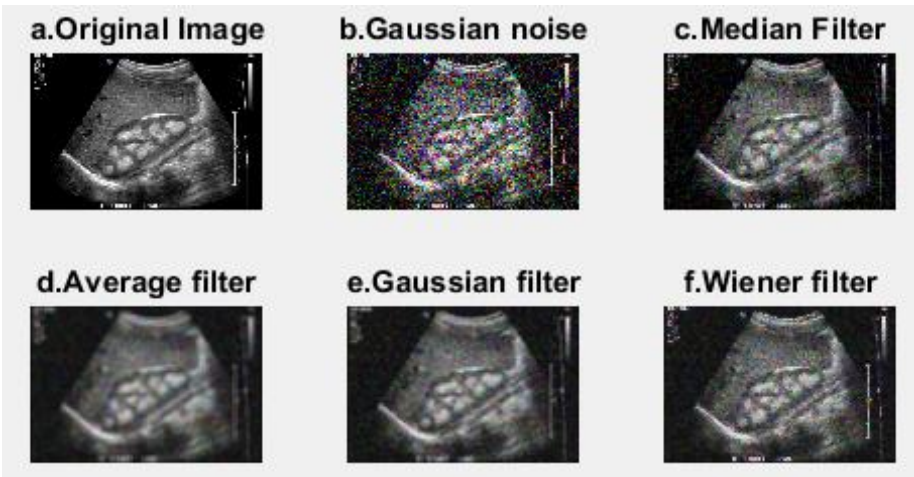

Fig.1. a) Original image b) Adding Gaussian noise and de-noising by c) Gaussian Filter d) Median Filter e) Average/Mean Filter f) Wiener Filter

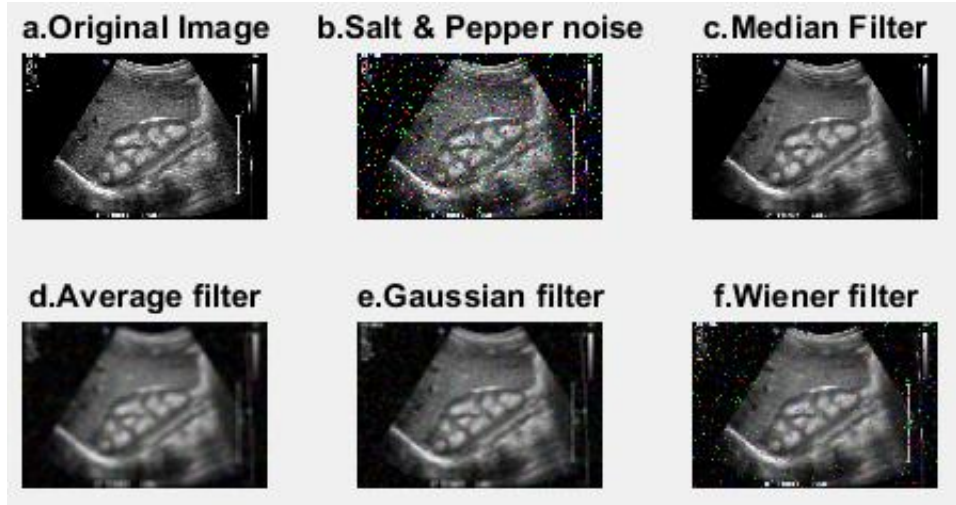

Fig.2. a) Original image b) Simulated Salt \& Pepper noise and de-noising by c) Gaussian Filter d) Median Filter e) Average/Mean Filter f) Wiener Filter
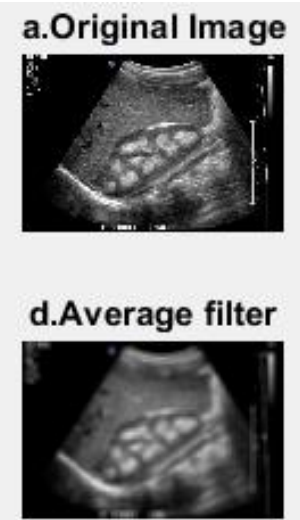
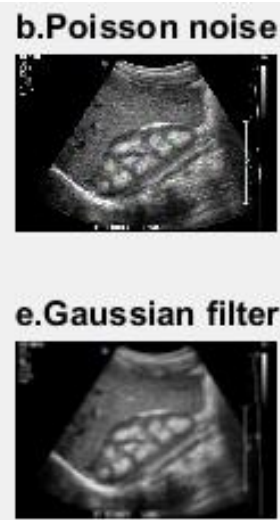

\section{c.Median Filter}

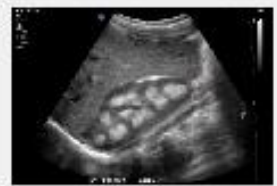

f.Wiener filter

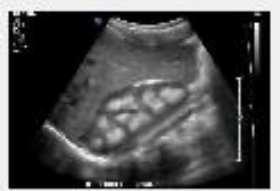

Fig.3. a) Original image b) Simulated Poisson noise and de-noising by c) Gaussian Filter d) Median Filter e) Average/Mean Filter f) Wiener Filter 


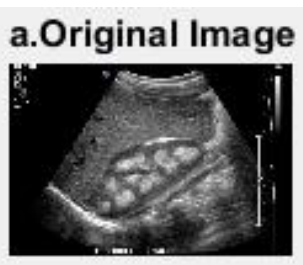

d.Average filter

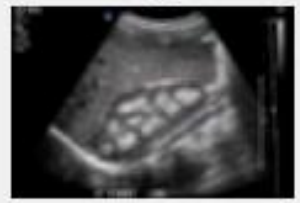

b.Speckle noise

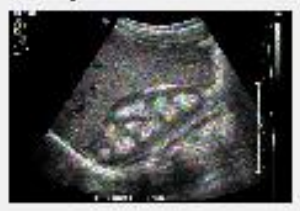

e.Gaussian filter

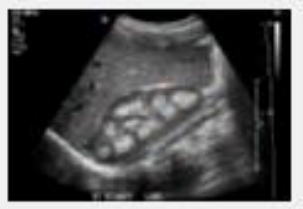

c.Median Filter

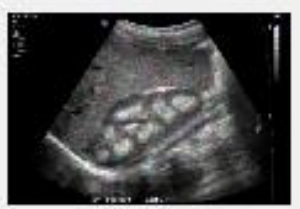

f.Wiener filter

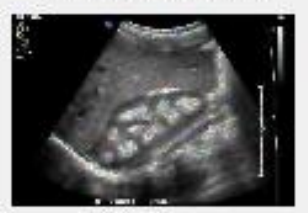

Fig.4. a) Original image b) Simulated Speckle noise and de-noising c) Gaussian Filter d) Median Filter e) Average/Mean Filter f) Wiener Filter

The comparison of images to achieve the most effective filter on different noises in different densities has been evaluated by peak signal-to-noise ratio (PSNR), Mean Squared Error (MSE) which is the well-known index to compare the original and de-noised image. Mostly, the higher PSNR and lower MSE conduct a higher quality and less noisy image. The following table shows the PSNR and MSEs for sample Stoned Kidney image which has been simulated by Gaussian, Salt \& Pepper, Speckle and Poisson noise, and then de-noised using Median filter, Mean /average filter, Gaussian Filter and Wiener filter to compare the performance for removing the noise and choosing the most effective filter.

Table 1 Performance of Filtering Methods for Gaussian Noise

\begin{tabular}{|c|c|c|c|}
\hline Sr. No & Filtering Method & MSE & PSNR \\
\hline 1 & Median filter & 2233.1220 & 14.6417 \\
\hline 2 & Mean /average filter & 2328.5984 & 14.4599 \\
\hline 3 & Gaussian Filter & 2198.6604 & 14.7092 \\
\hline 4 & Wiener filter & 1397.1111 & 16.6785 \\
\hline
\end{tabular}

According to table 1, Weiner filter has lowest MSE and Highest PSNR for Gaussian noise so wiener filter has best performance for Gaussian noise.

Table 2 Performance of Filtering Methods for Salt \& Pepper Noise

\begin{tabular}{|c|c|c|c|}
\hline Sr. No & Filtering Method & MSE & PSNR \\
\hline 1 & Median filter & 1570.5313 & 16.1703 \\
\hline 2 & Mean /average filter & 1698.9536 & 15.8290 \\
\hline 3 & Gaussian Filter & 1559.2654 & 16.2016 \\
\hline 4 & Wiener filter & 611.6919 & 20.2655 \\
\hline
\end{tabular}

According to table 2, Weiner filter has lowest MSE and Highest PSNR for Salt and Pepper noise so wiener filter has best performance for Salt and Pepper noise.

Table 3 Performance of Filtering Methods for Poisson Noise

\begin{tabular}{|c|c|c|c|}
\hline Sr. No & Filtering Method & MSE & PSNR \\
\hline 1 & Median filter & 379.5207 & 22.3384 \\
\hline 2 & Mean /average filter & 558.3726 & 20.6616 \\
\hline 3 & Gaussian Filter & 448.2516 & 21.6156 \\
\hline 4 & Wiener filter & 131.2176 & 26.9509 \\
\hline
\end{tabular}

According to table 3, Wiener filter has lowest MSE and Highest PSNR for Poisson noise so wiener filter has best performance for Poisson noise.

Table 4 Performance of Filtering Methods for Speckle Noise

\begin{tabular}{|c|c|c|c|}
\hline Sr. No & Filtering Method & MSE & PSNR \\
\hline 1 & Median filter & 594.5428 & 20.3890 \\
\hline 2 & Mean /average filter & 759.8717 & 19.3234 \\
\hline 3 & Gaussian Filter & 654.9647 & 19.9686 \\
\hline 4 & Wiener filter & 219.5729 & 24.7150 \\
\hline
\end{tabular}


According to table 4, Weiner filter has lowest MSE and Highest PSNR for Speckle noise so wiener filter has best performance for Speckle noise.

By analyzing four tables, we see the wiener filter has the best performance for four different types of noise for stoned kidney image.

\section{Conclusion}

In this paper, we have discussed four different filters and four kinds of noises (Speckle, Gaussian, Poisson and Salt \& Pepper) which are added to the stoned kidney image with different intensities for the application in medical field. The degraded image is de-noised by Median filter, Mean /average filter, Gaussian Filter and Wiener filter. The purpose is performing the comparison by calculating PSNR to figure out the behavior of filters in the presence of different kinds of noise. The results show the best performance of wiener filter in different intensities of Gaussian, Poisson. Salt and Pepper, and Speckle noise. In this paper PSNR and MSE has been used as comparison parameters. Results have been simulated on MATLAB 2015. Since the purpose of this paper is to give the idea to researchers for selecting the best techniques in the preprocessing of their kidney stone detection system to provide a desirable result, in future work we would like to perform further comparison in different wavelet-based techniques on stoned kidney images and evaluate the efficiency using the results in further stages of detection system.

\section{References}

[1]. Rafael C. Gonzalez and Richard E. Woods, "Digital Image Processing", Pearson Education, Second Edition, 2005.

[2]. Priyanka Rastogi and Neelesh Gupta " Review of Noise Removal Techniques for Fixed Valued Impulse Noise" , International Journal of Computer Applications (0975 - 8887) Volume 123 - No.5, August 2015

[3]. P.S. Hiremath, Prema T. Akkasaligar and Sharan Badiger, "Speckle Noise Reduction in Medical Ultrasound Images".

[4]. Pitas I, Venetsanopoulos A," Nonlinear mean filters in image processing", IEEE Transactions on Acoustics, Speech and Signal Processing, Volume:34, Issue: 3 , 573 - 584, Jun 1986.

[5]. Sarita D, "De-noising Techniques - A Comparison”, B.E., Andhra University College of Engineering, Visakhapatnam, India, 2000.

[6]. Suresh Kumar, Papendra Kumar, Manoj Gupta, Ashok Kumar Nagawat, "Performance Comparison of Median and Wiener Filter in Image De-noising", International Journal of Computer Application, Vol.12 - No.4, November 2010.

[7]. R.C. Gonzalez and R.E.Woods"Digital Image Processing."

[8]. Maria P, Costas P, “Image Processing: The Fundamentals”, Second edition, ISBN 978-0-470-74586-1, 2010.

[9]. Shi Zhong, Vladimir Cherkassky, “ Image De-noising using Wavelet Thresholding and Model Selection ”, IEEE, Proceedings 2000

[10]. Mengqi L, "Research on Image De-Noising Enhancement", Savonia University of Applied Sciences, Bachelor's thesis, September 2011.

[11]. Pawan P, Manoj G, Sumit S, Ashok KN," Image De-noising by Various Filters for Different Noise", International Journal of Computer Applications (0975 - 8887), Volume 9- No.4, November 2010.

[12]. Radhika V, Padmayathi G," A study on impulse noise removal for varied noise densities", Proceedings of the 1st Amrita ACM-W Celebration on Women in Computing in India, September 2010.

[13]. Ben W," Fast median and bilateral filtering", SIGGRAPH 2006 Papers, ACM, July 2006.

[14]. Gajanand G, "Algorithm for Image Processing Using Improved Median Filter and Comparison of Mean, Median and Improved Median Filter", International Journal of Soft Computing and Engineering (IJSCE) ISSN: 2231-2307, Volume-1, Issue-5, November 2011.

[15]. J. Chen, A. K. Jain, \A Structural Approach to Identify Defects on Textural Images\, Proceedings of the IEEE International Conference on Systems, Man, and Cybernetics, pp. 29-32, 1988.

[16]. Pitas I, Venetsanopoulos A," Nonlinear mean filters in image processing”, IEEE Transactions on Acoustics, Speech and Signal Processing, Volume:34, Issue: 3 , 573 - 584, Jun 1986.

[17]. Jiang JP, Yuan YT, Bao CP, “ The algorithm of fast filtering”, International Conference on Wavelet Analysis and Pattern Recognition, ICWAPR,2007.

[18]. Pei YH; Shin SC; Feng CH, “Generic 2D Gaussian smoothing filter for noisy image processing”,IEEE Region 10 Conference ( TENCON), 2007.

[19]. Mengqi L, "Research on Image De-Noising Enhancement", Savonia University of Applied Sciences, Bachelor's thesis, September 2011.

[20]. O. Laligant, GARCIA Frederic, IWiener filtering Image Analysis \.

[21]. Darcy Tsai, IGraduate Institute of Electronics Engineering,Nation Taiwan University,Taipei,Taiwan,ROC,Wiener Filters $\backslash$.

[22]. A. Vijayalakshmi, C. Titus and H. Lilly Beaulah, "Image Denoising for different noise models by various filters: A Brief Survey", International Journal of Emerging Trends \& Technology in Computer Science (IJETTCS) Web Site: www.ijettcs.org Email: editor@ijettcs.org, ISSN 2278-6856, vol. 3, Issue 6, (2014) November-December. 